\title{
Engineering of gold surface work function by electrodeposition of spirobifluorene donor-acceptor bipolar systems
}

\author{
Philipp Zabel ${ }^{\mathrm{a}}$, Thomas Dittrich ${ }^{\mathrm{a}, *}$, Yuan-Li Liao ${ }^{\mathrm{b}},{\text { Chi-Yen } \text { Lin }^{\mathrm{b}}{ }^{\mathrm{b}} \text {, Ken-Tsung Wong }}^{\mathrm{b}, *}$, \\ Fernando Fungo ${ }^{c, *}$, Luciana Fernandez ${ }^{c}$, Luis Otero ${ }^{c}$ \\ ${ }^{a}$ Helmholtz-Centre for Materials and Energy, Glienicker Str. 100, D-14109 Berlin, Germany \\ ${ }^{\mathrm{b}}$ Department of Chemistry, National Taiwan University, Taipei 106, Taiwan, ROC

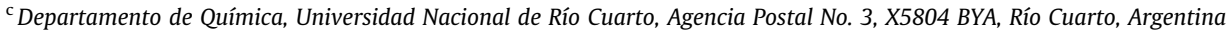

\section{A R T I C L E I N F O}

\section{Article history:}

Received 6 June 2009

Received in revised form 11 July 2009

Accepted 12 July 2009

Available online xxxx

\section{PACS:}

72.80.Le

73.20.-r

79.60.-i

82.45.Wx

Keywords:

Work function

Electropolymerization

Acceptor-donor polymer

\begin{abstract}
A B S T R A C T
Charge separation in gold/spirobifluorene-based donor (triphenylamine)-acceptor (cyano) bipolar systems has been investigated by contact potential difference and surface photovoltage spectroscopy. Organic films were formed on gold electrode by electrochemical polymerization and by dipping in dye solution. The gold surface work function has been changed over more than $0.4 \mathrm{eV}$ depending on the numbers of electrochemical deposition cycles. Photovoltage effects were analyzed in terms of internal photoemission from Au into organic film, and organic layer light absorption. The energetic differences between the Fermi-level of Au and HOMO levels of organic systems were obtained. The results showed that the electrodeposition is a versatile tool for electrode surface work function tuning.
\end{abstract}

(C) 2009 Elsevier B.V. All rights reserved.

\section{Introduction}

Interfaces between metals and organic materials are crucial for electronic properties and charge separation in devices such as organic light emitting diodes; organic field effect transistors; electrochromic systems and organic solar cells. The efficiency of these heterojunction devices strongly depends on the charge injection process (of holes or electrons) between the metal electrodes and the active organic layers. Metal/organic material interface is one of the device parameters that more attention has attracted in the search of improvement devices performance [1], because the formation of the metal/organic junction leads to

\footnotetext{
* Corresponding authors.

E-mail addresses: dittrich@helmholtz-berlin.de (T. Dittrich), kenwong@ntu.edu.tw (K.-T. Wong), ffungo@exa.unrc.edu.ar (F. Fungo).
}

modification in important properties, such as metal work function and orbital energy of the organic layer. These properties have a remarkable role in the charge transport across the interface. The presence of oriented molecules with intrinsic dipole over contact surfaces produces changes in the metal work function [2-6], and the interface dipole can acts as an improvement route for charge injection [7,8]. For example, Cho and Tao [9] showed that fine tuning of $\mathrm{Ag}$ and $\mathrm{Al}$ work function by carboxylates self assembled monolayer has a noticeable influence on electroluminescent devices. Thus, charge selective layer systems with molecular links are of great interest for applied and fundamental research. The most prominent example is probably the realization of bulk-hetero-junction solar cells with donor and acceptor molecules [10]. Mechanisms of separation of photo-generated charge carriers can give a deeper insight into the behavior of 
molecular systems. For example, charge separation by internal photoemission and exciton dissociation were distinguished by surface photovoltage spectroscopy in porphyrin layers deposited on gold [11].

A possible strategy to engineer surface work functions is related to the deposition of oriented molecules with donor-acceptor pairs (D-A), in which the A and D units are strong electron-withdrawing and electron-donating moieties, respectively. The appropriate choices of the molecular geometry and the A and D units characteristics allows the control of the dipole direction and magnitude. Furthermore, it is possible to tune the metal work function using molecular structures that hold D-A moieties with the capacity to form electropolymerized films over the metal surface. The electrochemical polymerization permits fine control over thickness and polymer properties; allowing to obtain stable and reproducible organic films over metal electrode. In this way, Shi et al. recently showed the capability of electrochemical deposited poly(3,4-ethylenedioxythiophene) (PEDOT) derivatives in the tuning of gold and ITO work functions [12].

In this work, we report the changes on gold surface work function by electropolymeric spirobifluorene-based bipolar films obtained from the monomers shown in Fig. 1. Recently, has been reported the electro-optical properties of polymer thin films on conducting substrate obtained by electropolymerization of pendant electroactive triphenylamine (TPA) units in spirofluorene derivatives [13-18]. Also, endcapping polyflourenes with holetransporting moieties showed the improvement of the performance of OLEDs [19-22]. The spiro linkage improves the morphological stability of the materials while retaining their electrical properties [23]. Moreover, the perpendicular arrangement of the two molecular halves leads to a high steric demand of the resulting rigid structure, efficiently suppressing molecular interactions between the $\pi$-systems [23]. In the present case, the spiro configuration
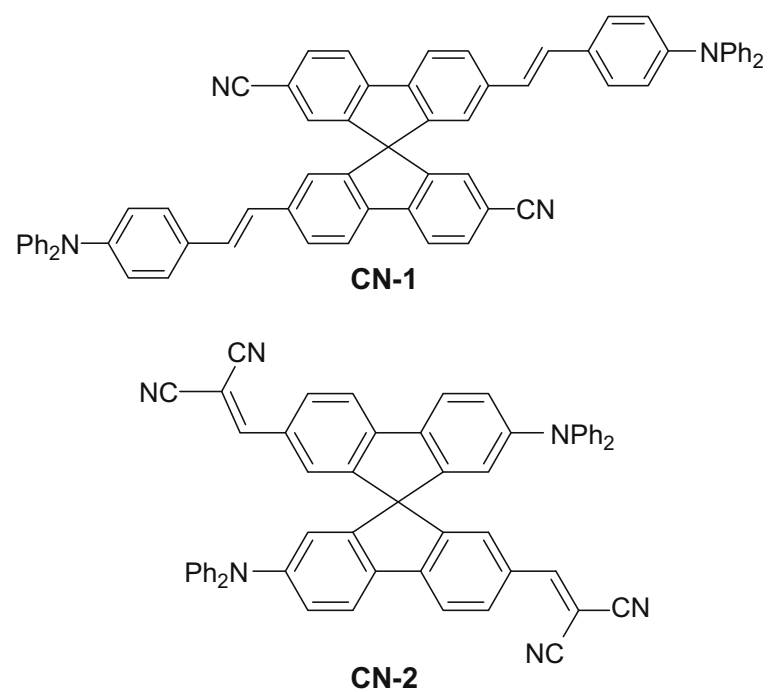

Fig. 1. Molecular structures of the spirobifluorene donor-acceptor bipolar systems. that bonds perpendicularly the two D-A moieties with a tetrahedral carbon impedes the $\pi$-orbital interaction between the different D-A substituents in the two molecular branches (Fig. 1). The electron-donating (D) moieties are TPA groups and the electron-withdrawing (A) are the cyano ones. In particular, CN1 molecules showed the capability to form stable electrogenerated polymeric films. Meanwhile CN2 is structurally related to CN1, but with higher electron acceptor capacity due to the presence of four cyano acceptor groups, and CN2 does not form an electropolymer due to the stability of its radical cation [18]. Here, the electrodeposited layers of CN1 were coated with CN2 by dipping in solution to investigate the role of the presence of an acceptor surface layer on the polymeric film. Changes of the surface work function and charge separation were studied by measurements of the contact potential difference (CPD) and surface photovoltage spectra (SPV) [24] in Kelvin-probe arrangement $[25,26]$ in order to distinguish charge separation mechanisms for different electronic transitions. It will be shown that spirobifluorene compounds with donor-acceptor pairs are suitable for engineering of the surface work function and open new opportunities in design and study of molecular architectures with applications in organic electronics.

\section{Experimental}

\subsection{General}

All the chemicals used where provided by SigmaAldrich in spectroscopic or electrochemical grade and used without purification. The synthesis and characterization of CN1 and CN2 molecules have been described elsewhere $[18,27]$. The CN1 polymer was obtained both, as film by electropolymerization over Au electrode, and in solution by chemical oxidation reaction [28].

\subsection{Sample preparation}

Layers of CN1 molecules were electro deposited on Au electrodes from $0.55 \mathrm{mM}$ in acetonitrile $(\mathrm{MeCN})$ solution containing $0.1 \mathrm{M}$ tetra $n$-butylammonium perchlorate (TBAP) as supporting electrolyte [18]. The Au electrodes were evaporated on glass coated with $\mathrm{Cr}$ as adhesion layer. The electro deposition was performed by cyclic voltammetry $(\mathrm{CV})$ at the scan rate of $0.1 \mathrm{~V} / \mathrm{s}$. A platinum coil was uses as counter electrode and a silver wire as a pseudo-reference electrode. Ferrocene was added to the cell as an internal standard. Fig. 2a shows 32 voltammograms of successive electro deposition of CN1 on a gold electrode. Upon oxidation, the TPA undergoes radical cation dimerization to produce tetraphenylbenzidine (TPB). The two TPA substituents act independently, in the two molecular branches, rendering the electropolymerization processes feasible $[17,18]$. The successive increase of the current indicates the formation of an electroactive film over the gold surface.

After rinsing in $\mathrm{MeCN}$ and exchanging the electrolyte to $0.1 \mathrm{M}$ TBAP in $\mathrm{MeCN}$, the electrodeposited layers of CN1 show the typical redox responses whereas the current density increases with the number of electro deposition cycles 


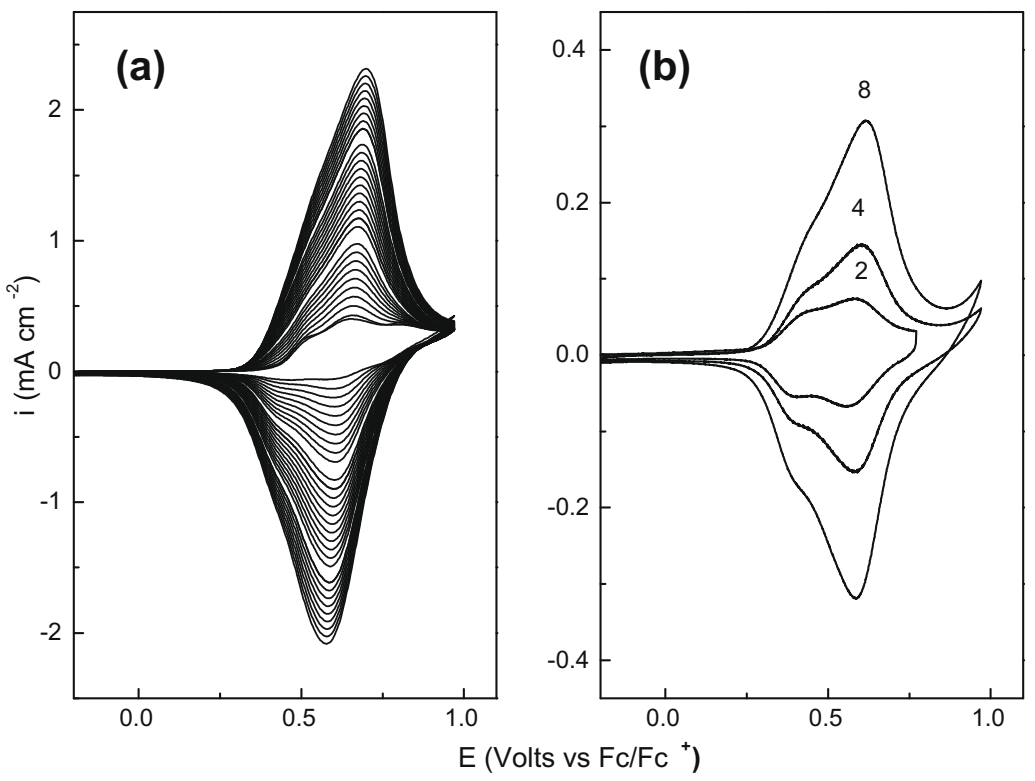

Fig. 2. (a) Successive cyclic voltammograms of CN1 electro deposition on Au electrode (0.55 mM in MeCN, $0.1 \mathrm{M}$ TBAP). (b) Cyclic voltammograms of electrodeposited CN1 grown at different potential cycle numbers (MeCN, 0.1 M TBAP).

(Fig. 2b). This behavior demonstrated the formation of stable layers of CN1 with different thickness. Each polymer sample was investigated before and after dipping in CN2 solution, in order to investigate the role of a surface layer with preferentially acceptor like character.

\subsection{Kelvin-probe and surface photovoltage spectroscop}

The contact potential difference was measured with a Kelvin-probe (Delta Phi Besocke) in a home-made vacuum chamber (pressure $2 \times 10^{-5} \mathrm{mbar}$ ). The same Kelvin-probe was used for investigations with surface photovoltage spectroscopy. The sample was illuminated through the vibrating gold mesh. A quartz prism monochromator (SPM2) with a halogen lamp was used for illumination in the photon energy range from 0.6 to $4 \mathrm{eV}$. The samples were stabilized in the dark before starting the surface photovoltage measurements. The scan rate of the surface photovoltage measurements was identical for all measurements. The accuracy of the absolute CPD signal is unknown due to the presence of uncontrolled adsorbates. We assumed that the relative influence of the adsorbates is the same for all samples. The measurement accuracies of the CPD and SPV signals were less than $1 \mathrm{mV}$. Transition energies were obtained with accuracies of the order of $10 \mathrm{meV}$.

\section{Results and discussion}

\subsection{Analysis of the contact potential difference}

Fig. 3 shows the dependence of the contact potential difference of $\mathrm{Au}$ electrodes coated with CN1 film on the number of electrochemical deposition cycles. The behavior of the contact potential difference describes the change of

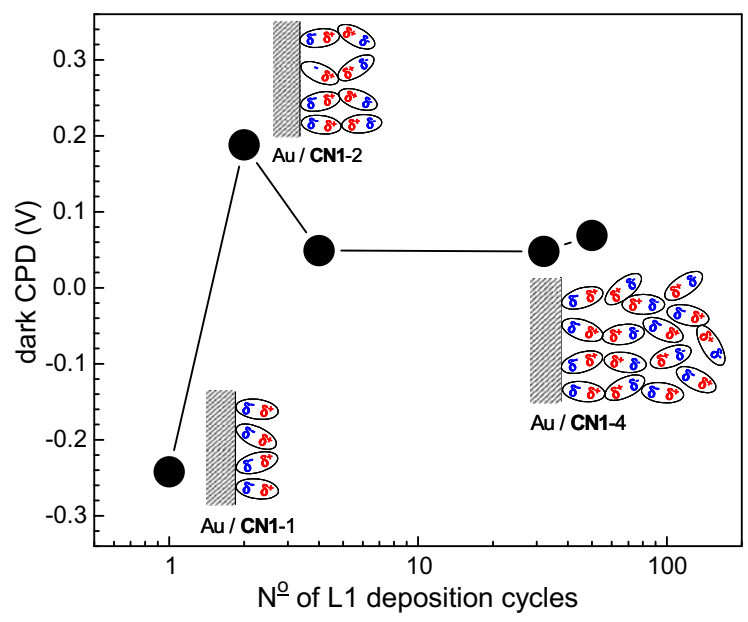

Fig. 3. Dependence of the contact potential difference of Au electrodes coated with $\mathbf{C N 1}$ film on the number of electrochemical deposition cycles.

the surface work function after layer deposition, i.e. the change of the surface dipole. The contact potential difference (CPD) of gold was set to $0 \mathrm{~V}$. The measurements of the CPD were performed in the dark after relaxation of the signal to a constant value. The CPD was about $-0.24 \mathrm{~V}$ after one electrochemical deposition cycle of CN1 molecules (CN1-1, Fig. 3). The value of CPD increased to about $0.2 \mathrm{~V}$ after two electrochemical deposition cycles (CN1-2) and remained nearly constant for more deposition cycles. The insets in Fig. 3 depict the idealized scheme of the orientation of CN1 molecules after one, two and more cycles of electro deposition. The lowest CPD was measured after one cycle which can be interpreted as a preferential orientation of $-\mathrm{CN}$ groups towards the gold surface. It is 
know the strong surface binding ability of the cyano nitrogen. Surface-enhanced Raman spectroscopy of a series of nitrile compounds [29-31] adsorbed on gold indicated the formation of $\sigma$-bonding of the nitrile nitrogen to the metal surface. Thus, it is not unlikely that CN1 monomer acquire a preferential orientation over electrode surface in the electropolymerization mechanism. We suggest that the cyano groups pick up electrons and link to the gold surface. On the other hand, the CPD jumped to its maximum after two deposition cycles of CN1. This can be explained in terms of preferential orientation of $-\mathrm{CN}$ groups towards the external surface. In this case, the $-\mathrm{NPh}_{2}$ groups of the first and second layer would bond each other during electropolymerization [18], so that the surface dipole changes the sign. For thick layers, CPD decreases to an average value. Obviously preferential orientation of surface dipoles is lost.

However, a layer of CN2 (which does not have electropolymerization capability) over naked gold surface obtained by dipping procedure shows small positive $\triangle \mathrm{CPD}$ effect (Fig. 4). The observed behaviors could be due to different molecular structural organization in the deposited layers. In the case of CN1 films, the polymerized structure can holds a preferential dipole orientation over gold surface, which is not present in the case of CN2 layer over naked gold. This shows the importance of electropolymerization procedure in the control of electrode work function. On the other hand, the value of CPD did not change after depositing CN2 molecules on the Au/CN1-1 sample (see Fig. 4). Therefore, the deposition of CN2 molecules on CN1 film by dipping does not change the preferential surface dipole orientation.

\subsection{Analysis of the surface photovoltage spectra}

The contact potential difference changed under illumination due to separation of photo-generated charge carriers. Contact potential difference spectra are shown in

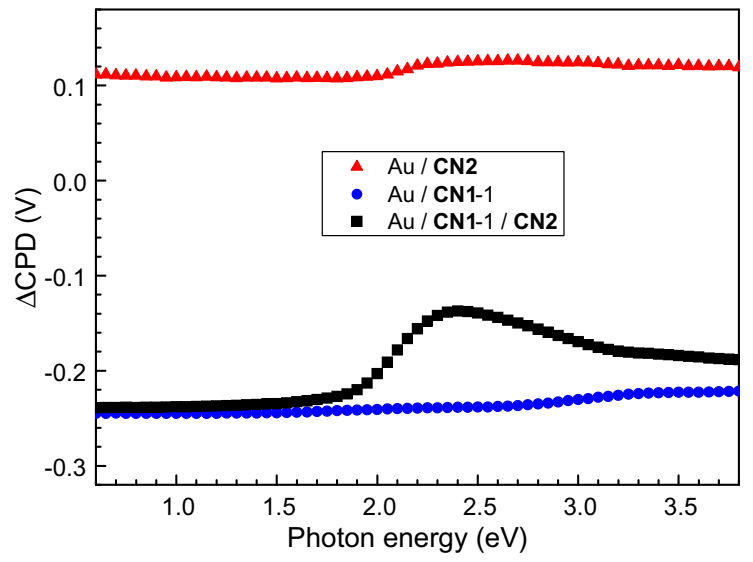

Fig. 4. Contact potential difference spectra under illumination of an $\mathrm{Au}$ electrode dipped in CN2 molecule solution ( $\mathrm{Au} / \mathrm{CN2}$ ) red triangles), an $\mathrm{Au}$ electrode with electrodeposited CN1 film after one electrochemical deposition cycle (Au/CN1-1, blue circles) and dipped in CN2 molecule solution (Au/CN1-1/CN2, black squares). (For interpretation of the references to colour in this figure legend, the reader is referred to the web version of this article.)
Fig. 4 for the $A u / C N 2, A u / C N 1-1$, and $A u / C N 1-1 / C N 2$ samples. A photoinduced increase of the contact potential difference means a preferential separation of photo-generated electrons (holes) towards the external (internal) surface whereas a photoinduced decrease of the contact potential difference means a preferential separation of photo-generated holes (electrons) towards the external (internal) surface. For the $\mathrm{Au} / \mathrm{CN2}$ and $\mathrm{Au} / \mathrm{CN1}-1$ samples the contact potential difference increases under illumination, i.e. electrons are separated preferentially towards the external surface. It should be noted, as is shown in Fig. 4, that the light induced change of the contact potential difference was quite small after electro deposition of layers of CN1 only, and clearly increased after dipping in CN2 solution. Remarkably CN2 over the naked gold surface shows only a small SPV effect.

Fig. 5 shows the (negative) surface photovoltage spectra of the $\mathrm{Au} / \mathrm{CN} 2, \mathrm{Au} / \mathrm{CN1}-1$, and $\mathrm{Au} / \mathrm{CN1}-1 / \mathrm{CN} 2$ samples together with the shapes of the optical density spectra of the CN1 polymer and CN2 molecules in diluted solution. For the Au/CN2 sample the SPV signal sets on at photon energy of $1.95 \mathrm{eV}$ (onset of the T1 transition). For comparison, the electronic transition sets on at a photon energy of $2.23 \mathrm{eV}$ for the optical density spectrum. Therefore, the electronic transitions of the SPV spectrum are red-shifted

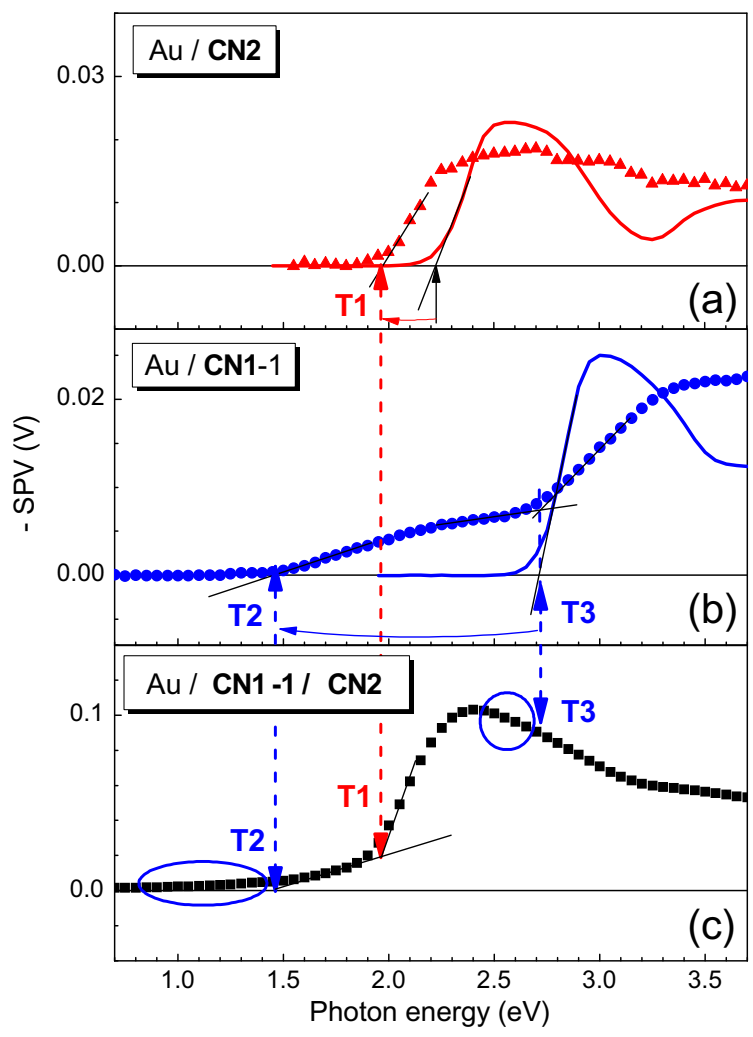

Fig. 5. Negative surface photovoltage spectra of $A u / C N 2$ (a), Au/CN1-1 (b) and Au/ CN1-1/ CN2 (c). The red and blue solid lines in (a) and (b) show the shapes of the optical density spectra of CN2 (a) and CN1 (b) molecules in solution. T1, T2 and T3 denote the onset of optical transitions. (For interpretation of the references to colour in this figure legend, the reader is referred to the web version of this article.) 
by $0.28 \mathrm{eV}$ in comparison to the optical density spectrum. The SPV signal remains nearly constant at photon energies higher than about $2.4 \mathrm{eV}$.

For the Au/CN1-1 sample two transitions (T2 and T3) can be distinguished in the SPV spectrum. The T2 and T3 transitions set on at a photon energies of 1.45 and $2.71 \mathrm{eV}$, respectively. For comparison, the electronic transition sets on at $2.71 \mathrm{eV}$ in the optical density spectrum. Therefore, the T3 and T2 transitions of the SPV spectrum correspond to the un-shifted and red-shifted by $1.26 \mathrm{eV}$ electronic transition of the optical density spectrum, respectively. The separations of photo-generated charge carriers lead always to the increase of the contact potential difference of the Au/CN1-1 sample.

All three transitions appear also in the SPV spectrum of the $\mathrm{Au} / \mathrm{CN1}-1 / \mathrm{CN2}$ sample as demonstrated in Fig. 4c. The SPV signals related to the $\mathrm{T} 1$ and $\mathrm{T} 2$ transitions have the same sign as for the $A u / C N 1-1$ and $A u / C N 2$ samples. At photon energies larger than $2.45 \mathrm{eV}$ the separation of photo-generated charge carriers lead to a decrease of the contact potential difference of the $\mathrm{Au} / \mathrm{CN1} 1-1 / \mathrm{CN} 2$ sample. The reason for this decrease is a change of the direction of separation of photo-generated charge carriers for the T3 transition.

The analysis of the SPV spectrum of the $\mathrm{Au} / \mathrm{CN1}-1 / \mathrm{CN2}$ sample shows that the T2 and T3 transitions are "broadened" towards lower photon energies, i.e. charge separation begins even at photon energies less than the onsets of the T2 and T3 transitions taken from the SPV spectrum of the $\mathrm{Au} / \mathrm{Au} / \mathrm{CN1}-1$ sample.

\subsection{Dependence of the $T 1, T 2$ and $T 3$ transitions on the CN1 layer thickness}

The SPV spectra did not change qualitatively if the thickness of the CN1 layer was increased by increasing the number of electrochemical deposition cycles. The detailed deconvolution of the SPV spectra is rather complicated since the spectra contain integrated and non-integrated components. For the discussion of the charge separation mechanisms a qualitative analysis will be sufficient. Therefore, here we describe the general tendencies of the thickness dependent SPV signals for the $\mathrm{T} 1, \mathrm{~T} 2$ and $\mathrm{T} 3$ transitions.

For the Au/CN1 samples the thickness dependencies of the SPV signals related to the T2 and T3 transitions can be distinguished if comparing the values of the SPV signals at 2.4 and $3.4 \mathrm{eV}$ (Fig. 5a). The (negative) SPV signal related to the T2 transition (value at $2.4 \mathrm{eV}$ ) increases with increasing number of electrodeposition cycles from $7 \mathrm{mV}$ $(\mathrm{Au} / \mathrm{CN1}-1)$ to $17(\mathrm{Au} / \mathrm{CN1}-2)$ and $33(\mathrm{Au} / \mathrm{CN1}-4) \mathrm{mV}$. For very large numbers of electrodeposition cycles the SPV signal related to the T2 transition decreases to 20 (Au/CN132 ) and $3(\mathrm{Au} / \mathbf{C N 1}-50) \mathrm{mV}$.

Contributions of the different transitions to the SPV signal can be estimated if considering differences of the SPV at characteristic energies where one or two transitions dominated the SPV spectrum. For the analysis we assume that the SPV signals of the various transitions are independent of each other and that the SPV signal related to the T2 transition tends to saturation at higher photon energies.
The (negative) SPV signal related to the T3 transition of the Au/CN1 samples can be described as the difference between the SPV signal obtained at 2.4 and $3.4 \mathrm{eV}$ (see also Fig. 6a). The SPV signals related to the T3 transition are 15, 16, $21 \mathrm{mV}$ for the Au/CN1-1, Au/CN1-2 and Au/CN1-4 samples, respectively what is nearly constant if comparing with the T2 transition. The SPV signal of the T3 transition decreases very strongly to 3 and $1 \mathrm{mV}$ for the Au/CN1-32 and $\mathrm{Au} / \mathrm{CN1}$-50 samples, respectively.

The (negative) SPV signal of the T1 transition can be estimated from the SPV spectra of the Au/CN1/CN2 samples in comparison to the Au/CN1 samples if assuming a linear superposition. Fig. 6b shows the values of the SPV signals measured at $2.4 \mathrm{eV}$ for the $\mathrm{Au} / \mathrm{CN1} / \mathrm{CN2}$ samples together with the difference between these values and those values measured at $2.4 \mathrm{eV}$ for the $\mathrm{Au} / \mathrm{CN} 1$ samples. It can be seen that the SPV signal related to the T1 transition decreases continuously with increasing number of electrodeposition cycles from 96 (Au/CN1-1/CN2), 64 (Au/CN1-2) CN2), 54 (Au/CN1-4/CN2), 35 (Au/CN1-32/CN2), 23 (Au/ CN1-50/CN2) mV.

\section{4. (d) Mechanisms of charge separation}

The T1 and T2 transitions should be related to internal photoemission of photo-generated electrons from $\mathrm{Au}$ into

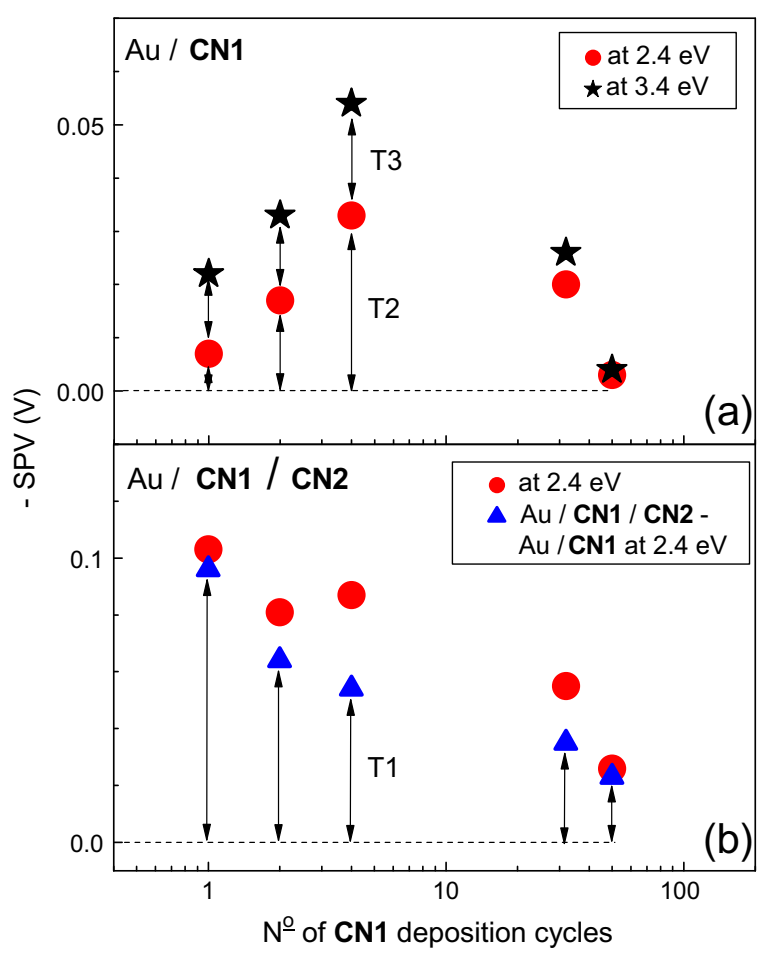

Fig. 6. Dependence of the negative values of the surface photovoltage on the number of electrochemical deposition cycles of CN1 molecules for $\mathrm{Au} /$ CN1 samples: (a) at 2.4 (red circles) and 3.4 (black stars) eV and of $\mathrm{Au}$ / CN1/CN2 samples (b) at $2.4 \mathrm{eV}$ (red circles) and of the difference of the surface photovoltages of the $\mathrm{Au} / \mathrm{CN1} / \mathrm{CN2}$ and $\mathrm{Au} / \mathrm{CN1}$ samples at $2.4 \mathrm{eV}$. (For interpretation of the references to colour in this figure legend, the reader is referred to the web version of this article.) 
the LUMO levels of the molecules (Fig. 7). In such case the red-shifted onsets of the transitions spectra in comparison to the optical density spectra correspond to the difference between the Fermi-level of the Au and the energy of the HOMO level of the respective molecule.

The SPV signal of the T1 transition decreased with increasing number of CN1 electrodeposition cycles. This can be understood with respect to Eq. (1).

$\mathrm{SPV}=\frac{\Delta d}{\varepsilon \cdot \varepsilon_{0}} \cdot \Delta Q$

A surface photovoltage signal contains information about the amount of charge separated in space $(\Delta Q)$ and about the distance over which the centres of positive and negative charges are separated $(\Delta d$, charge separation length) where $\varepsilon_{0}=8.85 \times 10^{-12} \mathrm{As} /(\mathrm{Vm})$ and $\varepsilon$ is the relative dielectric constant. Both parameters $\Delta Q$ and $\Delta d$ can be varied by changing the thickness of ultra-thin layers in charge selective layer systems. Thus, the charge separation length increases linearly with increasing number of CN1 electrodeposition cycles. However, the probability of internal photoemission of electrons from Au into the CN2 layer decreases exponentially with increasing distance. Therefore the overall SPV signal of the T1 transition decreases with increasing number of CN1 electrodeposition cycles (Fig. 6b).

Regarding to an exponential decrease of the charge transfer rate for internal photoemission with increasing distance, it is really surprising that the SPV signal of the $\mathrm{T} 1$ transition remains relatively large even for $\mathrm{Au} / \mathrm{CN1}$ 32/CN2 and Au/CN1-50/CN2 samples. We believe that prolonged electrodeposition of $\mathbf{C N} 1$ leads to the formation of porous or dendrite like structures giving space for quite inhomogeneous penetration of CN2 molecules during dipping. In order to investigate the film permeability, we performed $\mathrm{CV}$ with a model redox couple in solution (ferrocene), both over bare Au and Au/CN1 films. The ferrocene redox potential lies within the potential window where the CN1 film does not show an electrochemical response and, therefore, behaves solely as a blocking layer

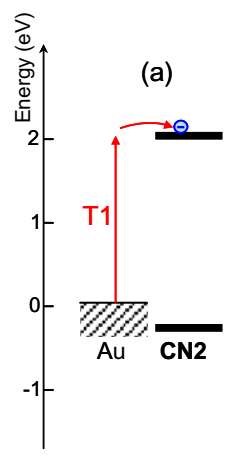

(b)

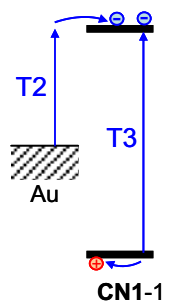

(c)

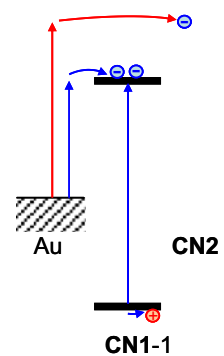

Fig. 7. Energy diagram of the $\mathrm{T} 1, \mathrm{~T} 2$ and $\mathrm{T} 3$ transitions in $\mathrm{Au} / \mathrm{CN2}$ (a), Au/ CN1-1 (b) and Au/ CN1-1/CN2 (c) samples. The transitions T1 and T2 are related to internal photoemission of electrons from the Au electrode into LUMO levels of the CN1 and CN2 molecules, the transitions T3 is related to light absorption in the CN1-1 film. on the Au electrode. If the polymer film forms an ideal compact layer, without defects or pinholes, not Faradaic current should be observed in the CV. However, in our case we obtained typical s-shape ultramicroelectrode voltammogram in the ferrocene oxidation over Au/CN1 film. As it is know, this kind of behavior is evidence of a high coverage of the Au bare electrode by a film with pores spaced far apart in relation to the radius of the pores [32]. This fact supports our assumption about CN2 molecules penetration during dipping process.

On the other hand, the SPV signal of the T2 transition increased with increasing number of CN1 electrodeposition cycles for CN1-1 to CN1-4 (Fig. 6a). This is not surprising since the amount of electrons emitted from $\mathrm{Au}$ to longer distances in the CN1 film increases with increasing number of CN1 electrodeposition cycles. However, the SPV signal of the T2 transition decreased for CN1-32 and CN150 layers. The origin of this effect is unclear, but could be due to morphological changes in the film with thickness increasing.

The SPV signal of the T3 transition has to be related to light absorption in the CN1 film. The (negative) SPV signal of the T3 transition of the Au/CN1 samples is practically constant for CN1-1, CN1-2 and CN1-4 since the charge separation length and the amount of photons absorbed in the first monolayer of CN1 molecules are practically unchanged. It means also that the ordering of the first CN1 monolayer at the Au surface is preserved during the following polymerization process. For CN1-32 and CN1-50 the amount of CN1 molecules in front of the first CN1 monolayer acts as an optical filter limiting strongly photon absorption in the CN1 monolayer at the Au surface leading to a strong decrease of the SPV signal. The (positive) SPV signal of the T3 transition in $\mathrm{Au} / \mathrm{CN1} / \mathrm{CN2}$ samples at energies values higher that $2.35 \mathrm{eV}$ is probably due to hole injection from CN1 excited state into CN2 molecules.

\section{Conclusions}

It has been shown that the work function of conducting surfaces can be engineered by electrodeposition of spirobifluorene compounds with donor-acceptor pairs. It should be mentioned that a modification of the work function of organic polymers is also possible due to electrochemically driven oxidation/reduction (for example, in PEDOT:PSS [33]). Related effects may disturb changes of the work function and charge separation caused by changes of preferential orientation of electrodeposited molecules. Some advantages of the used spirobifluorene derivatives are their synthetic versatility, stability and charge transport capability. The results showed that the formation of electrosynthetic films is a versatile tool for surface work function tuning. Surface dipole of a gold layer can be changed over more than $0.4 \mathrm{~V}$ by electrodeposition of CN1 molecules, and the surface dipole can be modified by internal photoemission. The highest SPV was reached after the combination of CN1 electrodeposited film and coated with CN2 molecule layer. This might become interesting for molecular electronics. 


\section{Acknowledgements}

The work has been supported by the DAAD-MINCYT grant number DA0601. We also thank to Consejo Nacional de Investigaciones Científicas y Técnicas (CONICET-Argentina), Agencia Nacional de Promoción Científica y Tecnológica (ANPCYT-Argentina), Secretaría de Ciencia y Técnica de la Universidad Nacional de Río Cuarto (SECYTUNRC).

\section{References}

[1] X. Crispin, V. Geskin, A. Crispin, J. Cornil, R. Lazzaroni, W.R. Salaneck, J.-L. Brédas, J. Am. Chem. Soc. 124 (2002) 8131.

[2] T. Nakamura, E. Koyama, Y. Shimoi, S. Abe, T. Ishida, K. Tsukagoshi, W. Mizutani, H. Tokuhisa, M. Kanesato, I. Nakai, H. Kondoh, T. Ohta, J. Phys. Chem. B 110 (2006) 9195.

[3] M.P. Nikiforov, U. Zerweck, P. Milde, Ch. Loppacher, T.-H. Park, H.T. Uyeda, M.J. Therien, L. Eng, D. Bonnell, Nano Lett. 8 (2008) 110

[4] R. Rosseau, V. De Renzi, R. Mazarello, D. Marchetto, R. Biagi, S. Scandolo, U. Del Pennino, J. Phys. Chem. B 110 (2006) 10862.

[5] N. Gozlan, U. Tisch, H. Haick, J. Phys. Chem. C 112 (2008) 12988.

[6] K. Homg, L.W. Lee, S.Y. Yang, K. Shin, H. Jeon, S.H. Kim, C. Yang, C.E. Park, Org. Electron. 9 (2008) 21.

[7] B.H. Hamadani, D.A. Corley, J.W. Ciszek, J.M. Tour, D. Nateson, Nano Lett. 6 (2006) 1303.

[8] C.-C. Hsiao, C.-H. Chang, H.-H. Lu, S.-A. Chen, Org. Electron. 8 (2007) 343.

[9] C.-P. Cho, Y.-T. Tao, Langmuir 23 (2007) 7090.

[10] N.S. Sariciftci, L. Smilowitz, A.J. Heeger, F. Wudl, Science 258 (1992) 1474.

[11] Y. Zidon, Y. Shapira, Th. Dittrich, L. Otero, Phys. Rev. B 75 (2007) 195327.

[12] Y. Shi, S.-C. Luo, W. Fang, K. Zhang, E.M. Ali, F.Y.C. Boey, J.Y. Ying, J. Wang, H.-H. Yu, L.-J. Li, Org. Electron. 9 (2008) 859

[13] K.-T. Wong, Y.-H. Lin, H.-H. Wu, F. Fungo, Org. Lett. 9 (2007) 4531.
[14] S. Lu, T. Liu, L. Ke, D.G. Ma, S.-J. Chua, W. Huang, Macromolecules 38 (2005) 8494.

[15] T. Oyamada, C.-H. Chang, T.-C. Chao, F.-C. Fang, C.-C. Wu, K.-T. Womg, H. Sasabe, C. Adachi, J. Phys. Chem. C 111 (2007) 111.

[16] C.-C. Wu, W.-G. Liu, W.-Y. Hung, T.-L. Liu, Y.-T. Lin, H.-W. Lin, K.-T Womg, Y.-Y. Chien, R.-T. Chen, T.-H. Hung, T.-C. Chao, Y.-M. Chen, Appl. Phys. Lett. 87 (2004) 052103.

[17] J. Natera, L. Otero, L. Sereno, F. Fungo, N.-S. Wang, Y.-M. Tsai, T.-Y. Hwu, K.-T. Wong, Macromolecules 40 (2007) 4456.

[18] L. Otero, L. Sereno, F. Fungo, Y.-L. Liao, C.-Y. Lin, K.-T. Wong, Chem. Mater. 18 (2006) 3495

[19] J.P.J. Markham, E.B. Namdas, T.D. Anthopoulos, I.D.W. Samuel, G.J. Richards, P.L. Burn, Appl. Phys. Lett. 85 (2004) 1463.

[20] W.-Y. Lai, R. Zhu, Q.-L. Fan, L.-T. Hou, Y. Cao, W. Huang, Macromolecules 39 (2006) 3707.

[21] P.A. Levermore, R. Xia, W. Lai, X.H. Wang, W. Huang, D.D.C. Bradley, J. Phys. D: Appl. Phys. 40 (2007) 1896.

[22] Z. Li, C. Di, Z. Zhu, G. Yu, Z. Li, Q. Zeng, Q. Li, Y. Liu, J. Qin, Polymer 47 (2006) 7889

[23] T.P.I. Saragi, T. Spehr, A. Siebert, Th. Fuhrmann-Lieker, J. Salbeck, Chem. Rev. 107 (2007) 1011.

[24] I. Mora-Seró, Th. Dittrich, G. García-Belmonte, J. Bisquert, J. Appl. Phys. 100 (2006) 103705.

[25] L. Kronik, Y. Shapira, Surf. Sci. Rep. 37 (1999) 1

[26] Th. Dittrich, S. Bönisch, P. Zabel, S. Dube, Rev. Sci. Instrum. 79 (2008) 113903.

[27] C.-L. Chiang, C.-F. Shu, C.-T. Chen, Org. Lett. 7 (2005) 3717.

[28] Chemical polymerization synthesis and characterization will be published elsewhere.

[29] P. Gao, M.J. Weaver, J. Phys. Chem. 89 (1985) 5040.

[30] D.S. Corrigan, P. Gao, L.-W.H. Leung, M.J. Weaver, Langmuir 2 (1986) 744.

[31] I.C.N. Diógenes, I.M.M. De Carvalho, E. Longhnotti, L.G.F. Lopes, L.M.A. Temperini, G.F.S. Andrade, I.S. Moreira, J. Electroanal. Chem. 605 (2007) 1.

[32] A.J. Bard, in: Integrated Chemical Systems, Wiley, New York, 1994, p. 196 (Chapter 5).

[33] See, for example A. Petr, F. Zhang, H. Peisert, M. Knupfer, L. Dunsch, Chem. Phys. Lett. 385 (2004) 140. 